\title{
Finite volume and partially quenched QCD-like effective field theories
}

\author{
Johan Bijnens and Thomas Rössler \\ Department of Astronomy and Theoretical Physics, Lund University, \\ Sölvegatan 14A, SE 223-62 Lund, Sweden \\ E-mail: bijnens@thep.lu.se, thomas.roessler@thep.lu.se
}

Abstract: We present a calculation of the meson masses, decay constants and quarkantiquark vacuum expectation value for the three generic QCD-like chiral symmetry breaking patterns $\mathrm{SU}\left(N_{F}\right) \times \mathrm{SU}\left(N_{F}\right) \rightarrow \mathrm{SU}\left(N_{F}\right)_{V}, \mathrm{SU}\left(N_{F}\right) \rightarrow \mathrm{SO}\left(N_{F}\right)$ and $\mathrm{SU}\left(2 N_{F}\right) \rightarrow$ $\operatorname{Sp}\left(2 N_{F}\right)$ in the effective field theory for these cases. We extend the previous two-loop work to include effects of partial quenching and finite volume.

The calculation has been performed using the quark flow technique. We reproduce the known infinite volume results in the unquenched case. The analytical results can be found in the supplementary material.

Some examples of numerical results are given. The numerical programs for all cases are included in version 0.54 of the CHIRON package.

The purpose of this work is the use in lattice extrapolations to zero mass for QCD-like and strongly interacting Higgs sector lattice calculations.

Keywords: Lattice Gauge Field Theories, Chiral Lagrangians, Technicolor and Composite Models, Effective field theories

ARXIV EPRINT: 1509.04082 


\section{Contents}

1 Introduction $\quad 1$

2 Quark level 3

2.1 The three Dirac fermion cases 3

2.2 Majorana fermions in a real representation 4

3 Effective field theory 5

3.1 The general LO and NLO Lagrangian 5

3.2 The three Dirac fermion cases 5

3.3 Majorana fermions in a real representation 7

4 Relation Dirac and Majorana for the adjoint case $\quad 8$

5 Partially quenching and the quark flow technique 9

6 Analytical results $\quad 10$

$\begin{array}{lll}7 & \text { Numerical examples and checks } & 13\end{array}$

8 Conclusions 14

\section{Introduction}

Effective field theory is used extensively in the study of strongly interacting gauge theories. A recent review covering a number of different applications in addition to other methods is [1]. Besides general interest in understanding strongly interacting gauge theories, they might still be useful as an alternative for the Standard Model Higgs sector as well as for dark matter. These applications have been reviewed recently at the 2015 [2, 3] and 2013 [4] lattice conferences. A number of recent lattice studies is [5-11]. Reviews of technicolor and strongly interacting Higgs sectors are [12-14].

Lattice studies are always performed at a nonzero fermion mass. In order to obtain results in the massless limit extrapolations are needed. A main tool for this in the context of lattice QCD is Chiral Perturbation Theory (ChPT) [15-17].

In the case of equal mass fermions three main symmetry breaking patterns are possible [18-20]. For $N_{F}$ Dirac fermions in a complex representation the global symmetry group is $\mathrm{SU}\left(N_{F}\right)_{L} \times \mathrm{SU}\left(N_{F}\right)_{R}$ and it breaks spontaneously to the diagonal subgroup $\mathrm{SU}\left(N_{F}\right)_{V}$. For $N_{F}$ Dirac fermions in a real representation the global symmetry group is $\mathrm{SU}\left(2 N_{F}\right)$ and it breaks spontaneously to $\mathrm{SO}\left(2 N_{F}\right)$. An alternative possibility is that we have $N_{F}$ 
Majorana fermions in a real representation with a global symmetry group $\mathrm{SU}\left(N_{F}\right)$ spontaneously broken to $\mathrm{SO}\left(N_{F}\right)$. We show in this work that the EFT for the quantities we consider is really the same as for Dirac fermions. The final case is $N_{F}$ Dirac fermions in a pseudo-real representation. The global symmetry group is again $\mathrm{SU}\left(2 N_{F}\right)$ but in this case it is expected to be broken spontaneously to $\operatorname{Sp}\left(2 N_{F}\right)$.

The effective field theory (EFT) for these cases is discussed at tree level or lowest order (LO) in [21]. At next-to-leading order (NLO) the first case is simply ChPT for $N_{F}$ light quarks with a symmetry breaking pattern of $\mathrm{SU}\left(N_{F}\right) \times \mathrm{SU}\left(N_{F}\right) \rightarrow \mathrm{SU}\left(N_{F}\right)$, a direct extension of the QCD case and was already done in [17]. The pseudo-real case was done at $\mathrm{NLO}$ by [22]. The $\mathrm{SU}\left(2 N_{F}\right) \rightarrow \mathrm{SO}\left(2 N_{F}\right)$ case was done in [23]. The extension for all three cases to next-to-next-to-leading order (NNLO) was done in earlier work by one of the authors [24]. More references to earlier work can be found there and in [25, 26].

This paper is an extension to the work of [24]. We add a short discussion showing that the calculations and the Lagrangian for the real case also covers the case of Majorana fermions in a real representation. The main part of the work concerns the extension of the calculations at NNLO order of the masses, decay constants and vacuum expectation values to include effects of partial quenching and finite volume.

Partial quenching was introduced in ChPT by [27]. A thorough discussion of the assumptions involved is in [28]. It allows to study a number of variations of input parameters at reduced cost, as discussed in e.g. [29]. We do not use the supersymmetric method introduced in [27] and extended (at NLO) to the cases discussed here in [23]. We only use the quark-flow technique introduced in [30]. Two-loop results in infinite volume partially quenched ChPT (PQChPT) for the masses and decay constants are in [31-33]. The definitions of the infinite volume integrals we use can be found there.

Finite volume effects in ChPT were introduced in ChPT in [34-36]. Early two-loop work is $[37,38]$. The vacuum expectation value was discussed in more detail in [39]. After the proper evaluation of the finite volume two-loop sunsetintegrals using two different methods [40] the masses and decay constants were treated in both the unquenched [41] and partially quenched [42] case. In particular the integral notation at finite volume we use is defined in [42].

In section 2 we recapitulate briefly the discussion from [24] at the quark level and add the case with Majorana fermions. Section 3 similarly recapitulates [24] at the effective field theory level and adds the Majorana fermion case. The cases with Dirac fermions and Majorana fermions are essentially identical from the EFT point of view for the quantities we consider. The underlying reason is an $\mathrm{U}\left(2 N_{F}\right)$ transformation that relates the two cases as discussed in section 4. Partial quenching and the quark flow techniques we have used for the different cases is discussed to some extent in section 5. For a discussion on finite volume and the notation used there we refer to [42]. Our analytical results are described in section 6, in particular we clarify the definitions of the decay constant and vacuum expectation value used in terms of quark fields. The numerical examples and checks are presented in section 7 . The analytical formulas are included in the supplementary file analyticalresults.txt (online resource 1) and the numerical programs are available via CHIRON, [43, 44]. The last section briefly recapitulates the main points of our work. 


\section{Quark level}

\subsection{The three Dirac fermion cases}

The discussion here is kept very short, longer versions can be found in [21] and [24]. This subsection is mainly included to show normalization conventions.

QCD or complex representation. In the $N_{F}$ equal mass Dirac fermions in a complex representation, we put the $N_{F}$ fermions together in an $N_{F}$ column matrix $q$. The global symmetry transformation by $g_{L} \times g_{R} \in \mathrm{SU}\left(N_{F}\right)_{L} \times \mathrm{SU}\left(N_{F}\right)_{R}$ is given by

$q_{L} \rightarrow g_{L} q_{L}, \quad q_{R} \rightarrow g_{R} q_{R}, l_{\mu} \rightarrow g_{L} l_{\mu} g_{L}^{\dagger}+i g_{L} \partial_{\mu} g_{L}^{\dagger}, \quad r_{\mu} \rightarrow g_{R} l_{\mu} g_{R}^{\dagger}+i g_{R} \partial_{\mu} g_{R}^{\dagger}, \mathcal{M} \rightarrow g_{R} \mathcal{M} g_{L}^{\dagger}$.

The matrix $\mathcal{M}=m_{q} \mathrm{I}+s+i p$ brings the quark mass term $m_{q} \mathrm{I}$ and the external scalar $s$ and pseudo-scalar densities in the Lagrangian via $-\bar{q}_{R} \mathcal{M} q_{L}+$ h.c.. The external fields $l_{\mu}, r_{\mu}$ are in the Lagrangian via $\bar{q}_{L} \gamma^{\mu} l_{\mu} q_{L}+\bar{q}_{R} \gamma^{\mu} r_{\mu} q_{R}$. Taking derivatives w.r.t. the external fields allows to calculate relevant Green functions $[16,17]$. In particular, deriving w.r.t. $s_{11}$ allows us to obtain $\left\langle\bar{q}_{L 1} q_{R 1}+\bar{q}_{R 1} q_{L 1}\right\rangle$ and derivatives w.r.t. $a_{\mu 12}$ with $r_{\mu 12}=-l_{\mu 12}=a_{\mu 12}$ allows access to matrix-elements of $\bar{q}_{2} \gamma^{\mu} \gamma^{5} q_{1}$ The symmetry is spontaneously broken by a vacuum expectation value

$$
\left\langle\bar{q}_{L j} q_{R i}\right\rangle=v_{0} \delta_{i j}
$$

This leaves a global symmetry $\mathrm{SU}\left(N_{F}\right)_{V}$ with $g_{L}=g_{R}$ unbroken.

Adjoint or real representation. When the fermions are in a real representation, we can introduce besides the $N_{F}$ right handed fermions $q_{R i}$ a second set of right handed fermions in the same gauge group representation, $\tilde{q}_{R i}=C \bar{q}_{L i}^{T}$. These can be put together in a $2 N_{F}$ column vector $\hat{q}, \hat{q}^{T}=\left(q_{R 1} \ldots q_{R N_{F}} \tilde{q}_{R 1} \ldots \tilde{q}_{R N_{F}}\right)$. The global symmetry transformation with $g \in \mathrm{SU}\left(2 N_{F}\right)$ is now

$$
\hat{q} \rightarrow g \hat{q}, \quad \hat{V}_{\mu} \rightarrow g \hat{V}_{\mu} g^{\dagger}, \quad \hat{\mathcal{M}} \rightarrow g \hat{\mathcal{M}} g^{T}
$$

We define the external densities and currents as in the QCD case with $r_{\mu}, l_{\mu}$ and $\mathcal{M}$. We define $2 N_{F} \times 2 N_{F}$ matrices

$$
\hat{\mathcal{M}}=\left(\begin{array}{cc}
0 & \mathcal{M} \\
\mathcal{M}^{T} & 0
\end{array}\right), \quad \hat{V}_{\mu}=\left(\begin{array}{cc}
r_{\mu} & 0 \\
0 & -l_{\mu}^{T}
\end{array}\right) .
$$

Note that the global symmetry can change quark-antiquark currents to diquark currents. The fermions condense forming a vacuum expectation value

$$
\frac{1}{2}\left\langle\hat{q}_{j}^{T} C \hat{q}_{j}\right\rangle=v_{0} J_{S i j} \quad J_{S}=\left(\begin{array}{cc}
0 & \mathrm{I} \\
\mathrm{I} & 0
\end{array}\right) .
$$

This leaves a global symmetry $\mathrm{SO}\left(2 N_{F}\right)$ with $g J_{S} g^{T}=J_{S}$. 
$N_{c}=\mathbf{2}$ or pseudo-real representation. When the fermions are in a pseudo-real representation, we can introduce besides the $N_{F}$ right handed fermions $q_{R i a}$ again a second set of right handed fermions in the same gauge group representation, $\tilde{q}_{R i a}=\epsilon_{a b} C \bar{q}_{L i b}^{T} . a, b$ are gauge indices and the extra Levi-Civita tensor $\epsilon_{a b}$ is needed to have $\tilde{q}_{R i a}$ transform under the gauge group as $q_{i R a}$. The explicit formula is for the case of the fundamnetal representation with $N_{c}=2 . q_{R i}$ and $\tilde{q}_{R i}$ can be put together in a $2 N_{F}$ column vector $\hat{q}$, $\hat{q}^{T}=\left(q_{R 1} \ldots q_{R N_{F}} \tilde{q}_{R 1} \ldots \tilde{q}_{R N_{F}}\right)$. The global symmetry transformation with $g \in \operatorname{SU}\left(2 N_{F}\right)$ is now

$$
\hat{q} \rightarrow g \hat{q}, \quad \hat{V}_{\mu} \rightarrow g \hat{V}_{\mu} g^{\dagger}, \quad \hat{\mathcal{M}} \rightarrow g \hat{\mathcal{M}} g^{T}
$$

We define the external densities and currents as in the QCD case with $r_{\mu}, l_{\mu}$ and $\mathcal{M}$. We then define

$$
\hat{\mathcal{M}}=\left(\begin{array}{cc}
0 & -\mathcal{M} \\
\mathcal{M}^{T} & 0
\end{array}\right), \quad \hat{V}_{\mu}=\left(\begin{array}{cc}
r_{\mu} & 0 \\
0 & -l_{\mu}^{T}
\end{array}\right) .
$$

Note that the global symmetry can again change quark-antiquark currents to diquark currents. The fermions condense forming a vacuum expectation value

$$
\frac{1}{2}\left\langle\hat{q}_{j a}^{T} \epsilon_{a b} C \hat{q}_{j b}\right\rangle=v_{0} J_{A i j} \quad J_{A}=\left(\begin{array}{cc}
0 & -\mathrm{I} \\
\mathrm{I} & 0
\end{array}\right) .
$$

This leaves a global symmetry $\operatorname{Sp}\left(2 N_{F}\right)$ with $g J_{A} g^{T}=J_{A}$.

\subsection{Majorana fermions in a real representation}

In the earlier work [24] at infinite volume Dirac fermions and Dirac masses were assumed. It was then also asumed that the vacuum condensate was aligned with the Dirac fermion masses. There is in fact another possibility. Majorana fermions with a Majorana mass in a real representation of the gauge group. In this case the global symmetry is $\mathrm{SU}\left(N_{F}\right)$. It is expected to be spontaneously broken down to $\mathrm{SO}\left(N_{F}\right)$ which is aligned with the Majorana masses.

A Majorana spinor is a Dirac spinor that satisfies

$$
\psi=C \bar{\psi}^{T} \quad \text { or } \quad \psi=\left(\begin{array}{c}
\psi_{M} \\
-i \sigma^{2} \psi_{M}^{*}
\end{array}\right) .
$$

The last equality are in the chiral representation for the Dirac matrices. The Lagrangian for a single free Majorana fermion is

$$
\frac{1}{2} \bar{\psi} i \gamma^{\mu} \partial_{\mu} \psi-\frac{m}{2} \bar{\psi} \psi=\psi_{M}^{\dagger} C i \bar{\sigma}^{\mu} \partial_{\mu} \psi-\frac{i m}{2}\left(\psi_{M}^{T} \sigma^{2} \psi+\psi_{M}^{\dagger} \sigma^{2} \psi^{*}\right)
$$

$\bar{\sigma}^{0}=\mathrm{I}, \bar{\sigma}^{i}=-\sigma^{i}$. If we want to gauge this for $m \neq 0$ the mass term requires the fermions to be in a real representation of the gauge group.

For $N_{F}$ Majorana fermions $\psi_{M i}$ in the adjoint representation with external fields $\hat{V}_{\mu}$ and $\hat{\mathcal{M}}$ the Lagrangian, put in a big column vector $\hat{q}^{T}=\left(\psi_{1}^{T} \ldots \psi_{N_{F}}^{T}\right)$ is

$$
\mathcal{L}=\operatorname{tr}_{c}\left(\hat{q}^{\dagger} \bar{\sigma}^{\mu}\left(i D_{\mu}+\hat{V}_{\mu}\right) \hat{q}\right)-\frac{i}{2} \operatorname{tr}_{c}\left(\hat{q}^{T} \sigma^{2} \hat{\mathcal{M}}^{\dagger} \hat{q}+\hat{q}^{\dagger} \sigma^{2} \hat{\mathcal{M}} \hat{q}^{*}\right)
$$


This Lagrangian has a global $\mathrm{SU}\left(N_{F}\right)$ symmetry with $g \in \mathrm{SU}\left(N_{F}\right)$ with

$$
\hat{q} \rightarrow g \hat{q}, \quad \hat{V}_{\mu} \rightarrow g \hat{V}_{\mu} g^{\dagger}+i g \partial_{\mu} g^{\dagger}, \quad \hat{\mathcal{M}} \rightarrow g \hat{\mathcal{M}} g^{T} .
$$

The maximal symmetry argument says that in this case the fermions will condense to the flavour neutral vacuum $\left\langle\operatorname{tr}_{c}\left(\hat{q}^{T} C \hat{q}\right)\right\rangle$. This is conserved by the part of the global group that satisfies $g g^{T}=\mathrm{I}$ or the conserved part of the global symmetry group is $\mathrm{SO}\left(N_{F}\right)$.

Note that the form of the vacuum and the form of the mass term are the only differences as far as the global symmetry group and its breaking are concerned compared to the case with $N_{F} / 2$ Dirac fermions in a real representation.

\section{$3 \quad$ Effective field theory}

\subsection{The general LO and NLO Lagrangian}

The ChPT Lagrangian for $N_{F}$ flavours at LO and NLO has been derived in [17]. The Lagrangian for the other cases has the same form as has been shown in [21,24] and other papers. The precise derivation can be found in [24] and the Majorana fermion case below in section 3.3.

In terms of the quantities $u_{\mu}, f_{ \pm \mu \nu}, \chi_{ \pm}$defined below for each case the lowest order Lagangian is

$$
\mathcal{L}_{2}=\frac{F^{2}}{4}\left\langle u_{\mu} u^{\mu}+\chi_{+}\right\rangle .
$$

Here we use the notation $\langle A\rangle=\operatorname{tr}_{F}(A)$, denoting the trace over flavours. The NLO Lagrangian derived by [17] reads

$$
\begin{aligned}
\mathcal{L}_{4}= & L_{0}\left\langle u^{\mu} u^{\nu} u_{\mu} u_{\nu}\right\rangle+L_{1}\left\langle u^{\mu} u_{\mu}\right\rangle\left\langle u^{\nu} u_{\nu}\right\rangle+L_{2}\left\langle u^{\mu} u^{\nu}\right\rangle\left\langle u_{\mu} u_{\nu}\right\rangle+L_{3}\left\langle u^{\mu} u_{\mu} u^{\nu} u_{\nu}\right\rangle \\
& +L_{4}\left\langle u^{\mu} u_{\mu}\right\rangle\left\langle\chi_{+}\right\rangle+L_{5}\left\langle u^{\mu} u_{\mu} \chi_{+}\right\rangle+L_{6}\left\langle\chi_{+}\right\rangle^{2}+L_{7}\left\langle\chi_{-}\right\rangle^{2}+\frac{1}{2} L_{8}\left\langle\chi_{+}^{2}+\chi_{-}^{2}\right\rangle \\
& -i L_{9}\left\langle f_{+\mu \nu} u^{\mu} u^{\nu}\right\rangle+\frac{1}{4} L_{10}\left\langle f_{+}^{2}-f_{-}^{2}\right\rangle+\frac{1}{2} H_{1}\left\langle f_{+}^{2}-f_{-}^{2}\right\rangle+\frac{1}{4} H_{2}\left\langle\chi_{+}^{2}-\chi_{-}^{2}\right\rangle .
\end{aligned}
$$

The NNLO Lagrangian has been classified for the $N_{F}$-flavour case in [45]. The Lagrangian at NNLO for the other cases is not known, the direct equivalent of the results in [45] is definitely a complete Lagrangian but might not be minimal. For this reason we do not quote the dependence on the NNLO Lagrangian in the real and pseudo-real cases.

The divergences at NLO were derived for the QCD case in [17], for the others in [22, 24]. At NNLO only the QCD case is known [46].

\subsection{The three Dirac fermion cases}

A more extensive discussion can be found in $[21,24]$. Here we simply quote the results.

When we have a global symmetry group $G$ with generators $T^{a}$ which is spontaneously broken down to a subgroup $H$ with generators $Q^{a}$ which form a subset of the $T^{a}$, the Goldstone bosons can be described by the $\operatorname{coset} G / H$. This coset can be parametrized [47, $48]$ via the broken generators $X^{a}$. Below we explain what is used for the different cases. We always work with generators normalized to 1 , i.e. $\left\langle X^{a} X^{b}\right\rangle=\delta^{a b}$.

The quantities used from the quark level are given in section 2. 
QCD or complex representation. The Goldstone boson manifold is in this case $\mathrm{SU}\left(N_{F}\right) \times \mathrm{SU}\left(N_{F}\right) / \mathrm{SU}\left(N_{F}\right)$ which itself has the structure of an $\mathrm{SU}\left(N_{F}\right)$ Note that the axial generators do not generate a subgroup of $\mathrm{SU}\left(N_{F}\right) \times \mathrm{SU}\left(N_{F}\right)$ even if $G / H$ has the structure of a group in this case.

We choose as the broken generators $X^{a}$ the generators of $\mathrm{SU}\left(N_{F}\right) \approx G / H$. The quantities needed to construct the Lagrangian and their symmetry transformations are

$$
\begin{aligned}
u & =\exp \left(\frac{i}{\sqrt{2} F} \pi^{a} X^{a}\right) \rightarrow g_{R} u h^{\dagger} \equiv h u g_{L}^{\dagger} \\
u_{\mu} & =i\left(u^{\dagger}\left(\partial_{\mu}-i r_{\mu}\right) u-u\left(\partial_{\mu}-l_{\mu}\right) u^{\dagger}\right) \rightarrow h u_{\mu} h^{\dagger}, \\
\chi & =2 B_{0} \mathcal{M} \rightarrow g_{R} \chi g_{L}^{\dagger} \\
\chi_{ \pm} & =u^{\dagger} \chi u^{\dagger} \pm u \chi^{\dagger} u \rightarrow h \chi_{ \pm} h^{\dagger}, \\
l_{\mu \nu} & =\partial_{\mu} l_{\nu}-\partial_{\mu} l_{\nu}-i l_{\mu} l_{\mu}+i l_{\nu} l_{\mu} \rightarrow g_{L} l_{\mu \nu} g_{L}^{\dagger} \\
r_{\mu \nu} & =\partial_{\mu} r_{\nu}-\partial_{\mu} r_{\nu}-i r_{\mu} r_{\mu}+i r_{\nu} r_{\mu} \rightarrow g_{R} r_{\mu \nu} g_{R}^{\dagger} \\
f_{ \pm \mu \nu} & =u l_{\mu \nu} u^{\dagger} \pm u^{\dagger} r_{\mu \nu} u \rightarrow h f_{ \pm \mu \nu} h^{\dagger} .
\end{aligned}
$$

The first line defines $h[47,48]$.

Adjoint or real representation. The Goldstone boson manifold is in this case $\mathrm{SU}\left(2 N_{F}\right)$ / $\mathrm{SO}\left(2 N_{F}\right)$. The unbroken generators satisfy $Q^{a} J_{S}=-J_{S} Q^{a T}$ which follows from $g J_{S} g^{T}=$ $J_{S}$. The broken generators satisfy $J_{S} X^{a}=X^{a T} J_{S}$.

The quantities needed to construct the Lagrangians are [24]

$$
\begin{aligned}
u & =\exp \left(\frac{i}{\sqrt{2} F} \pi^{a} X^{a}\right) \rightarrow g u h^{\dagger} \\
u_{\mu} & =i\left(u^{\dagger}\left(\partial_{\mu}-i \hat{V}_{\mu}\right) u-u\left(\partial_{\mu}+i J_{S} \hat{V}_{\mu}^{T} J_{S}\right) u^{\dagger}\right) \\
\chi & =2 B_{0} \hat{\mathcal{M}} \\
\chi_{ \pm} & =u^{\dagger} \chi J_{S} u^{\dagger} \pm u J_{S} \chi^{\dagger} u \\
\hat{V}_{\mu \nu} & =\partial_{\mu} \hat{V}_{\nu}-\partial_{\nu} \hat{V}_{\mu}-i\left(\hat{V}_{\mu} \hat{V}_{\nu}-\hat{V}_{\nu} \hat{V}_{\mu}\right) \\
f_{ \pm \mu \nu} & =J_{S} u \hat{V}_{\mu \nu} u^{\dagger} J_{S} \pm u \hat{V}_{\mu \nu} u^{\dagger}
\end{aligned}
$$

The first line defines $h$ by requiring that $g u h^{\dagger}$ is of the form $\exp \left(i \pi^{a} X^{a} /(\sqrt{2} F)\right)$. Note that the derivation used $J_{S} u=u^{T} J_{S}$.

$\boldsymbol{N}_{\boldsymbol{c}}=\mathbf{2}$ or pseudo-real representation. The Goldstone boson manifold is $\mathrm{SU}\left(2 N_{F}\right) /$ $\operatorname{Sp}\left(2 N_{F}\right)$. The unbroken generators satisfy $Q^{a} J_{A}=-J_{A} Q^{a T}$ which follows from $g J_{A} g^{T}=$ $J_{A}$. The broken generators satisfy $J_{A} X^{a}=X^{a T} J_{A}$. 
The quantities needed are [24]

$$
\begin{aligned}
u & =\exp \left(\frac{i}{\sqrt{2} F} \pi^{a} X^{a}\right) \rightarrow g u h^{\dagger} \\
u_{\mu} & =i\left(u^{\dagger}\left(\partial_{\mu}-i \hat{V}_{\mu}\right) u-u\left(\partial_{\mu}+i J_{A} \hat{V}_{\mu}^{T} J_{A}^{T}\right) u^{\dagger}\right), \\
\chi & =2 B_{0} \hat{\mathcal{M}} \\
\chi_{ \pm} & =u^{\dagger} \chi J_{A}^{T} u^{\dagger} \pm u J_{A} \chi^{\dagger} u \\
\hat{V}_{\mu \nu} & =\partial_{\mu} \hat{V}_{\nu}-\partial_{\nu} \hat{V}_{\mu}-i\left(\hat{V}_{\mu} \hat{V}_{\nu}-\hat{V}_{\nu} \hat{V}_{\mu}\right) \\
f_{ \pm \mu \nu} & =J_{A} u \hat{V}_{\mu \nu} u^{\dagger} J_{A}^{T} \pm u \hat{V}_{\mu \nu} u^{\dagger}
\end{aligned}
$$

The first line defines $h$ by requiring that $g u h^{\dagger}$ is of the form $\exp \left(i \pi^{a} X^{a} /(\sqrt{2} F)\right)$. Note that the derivation used $J_{A} u=u^{T} J_{A}$.

\subsection{Majorana fermions in a real representation}

The vacuum in this case is characterized by the condensate

$$
\frac{1}{2}\left\langle\hat{q}_{i}^{T} C \hat{q}_{j}\right\rangle=\frac{1}{2}\langle\bar{q} q\rangle \delta_{i j} .
$$

Under the symmetry group $g \in \mathrm{SU}\left(N_{F}\right)$ this moves around as

$$
\delta_{i j} \rightarrow\left(g^{T} g\right)_{i j}
$$

The unbroken part of the group is given by the generators $\tilde{Q}^{a}$ and the broken part by the generators $\tilde{X}^{a}$ which satisfy

$$
\tilde{Q}^{a}=-\tilde{Q}^{a T}, \quad \tilde{X}^{a}=\tilde{X}^{a T} .
$$

Just as in the cases discussed in [24] we can construct a rotated vacuum in general by using the broken part of the symmetry group on the vacuum. This leads to a matrix

$$
U=u u^{T} \rightarrow g U g^{T} \quad \text { with } \quad u=\exp \left(\frac{i}{\sqrt{2} F} \pi^{a} X^{a}\right)
$$

The matrix $u$ transforms as in the general $C C W Z$ case as

$$
u \rightarrow g u h^{\dagger} .
$$

Some earlier work used the matrix $U$ to describe the Lagrangian [21]. Here we will, as in [24] use the CCWZ scheme to obtain a notation that is formally identical to the QCD case. We add $N_{F} \times N_{F}$ matrices of external fields $\hat{V}_{\mu}$ and $\hat{\mathcal{M}}$. We need to obtain the $u_{\mu}$, or broken generator, parts of $u^{\dagger}\left(\partial_{\mu}-i V_{\mu}\right) u$. Eq. (3.8) have as a consequence that $u$ satisfies

$$
u=u^{T} .
$$


This leads using the same method as in [24] to

$$
u_{\mu}=i\left(u^{\dagger}\left(\partial_{\mu}-i \hat{V}_{\mu}\right) u-u\left(\partial_{\mu}+i \hat{V}_{\mu}^{T}\right) u^{\dagger}\right)
$$

With this we can construct Lagrangians. The equivalent quantities to the field strengths are

$$
f_{ \pm \mu \nu}=u \hat{V}_{\mu \nu} u^{\dagger} \pm u \hat{V}_{\mu \nu} u^{\dagger}
$$

with $\hat{V}_{\mu \nu}=\partial_{\mu} \hat{V}_{\nu}-\partial_{\nu} \hat{V}_{\mu}-i\left(\hat{V}_{\mu} \hat{V}_{\nu}-\hat{V}_{\nu} \hat{V}_{\mu}\right)$ and for the mass matrix

$$
\chi_{ \pm}=u^{\dagger} \chi u^{\dagger T} \pm u^{T} \chi^{\dagger} u
$$

with $\chi=2 B_{0} \hat{\mathcal{M}}$. The Lagrangians at LO and NLO have exactly the same form as given in (3.1) and (3.2) with $u_{\mu}, \chi_{ \pm}$and $f_{ \pm \mu \nu}$ as defined in (3.12), (3.13) and (3.14).

\section{Relation Dirac and Majorana for the adjoint case}

As discussed below, we have calculated the adjoint case using two methods. They were appropriate for the Dirac and the Majorana case respectively. After doing the trivial $2 N_{F} \rightarrow N_{F}$ change the results agreed exactly. If we compare the two cases, we see that the main difference is really the choice of vacuum.

The Dirac and Majorana cases lead to a choice of vacuum

$$
\left\langle\hat{q}_{i}^{T} C \hat{q}_{j}\right\rangle_{D} \propto J_{S i j}, \quad\left\langle\hat{q}_{i}^{T} C \hat{q}_{j}\right\rangle_{D} \propto \mathrm{I}_{i j}
$$

Is it possible to relate the two cases in a simple way? Under a global symmetry transformation the first one transforms as $J_{S} \rightarrow g J_{S} g^{T}$. If we could find a global transformation $g_{R}$ that lead to $g_{R} J_{S} g_{R}^{T}=$ I the two cases would be obviously the same.

It is not possible in general with a $\mathrm{SU}\left(2 N_{F}\right)$ rotation to accomplish this since $\operatorname{det} J_{S}=$ \pm 1 ( -1 for the $2 N_{F}=2$ ) while $\operatorname{det} \mathrm{I}=1$. However it is possible with a $\mathrm{U}(2 N)$ transformation. An explicit choice for $g_{R}$, with a free phase $\alpha$ is

$$
g_{R}=\frac{1}{\sqrt{2}}\left(\begin{array}{cc}
\mp i e^{i \alpha} \mathrm{I} & \pm i e^{-i \alpha} \mathrm{I} \\
e^{i \alpha} \mathrm{I} & e^{-i \alpha} \mathrm{I}
\end{array}\right) .
$$

It can be checked that this transforms a Dirac mass term for $N_{F}$ Dirac fermions into a Majorana mass term for $2 N_{F}$ Majorana fermions.

Inspections of the effective Lagrangians needed lead to the immediate conclusion that the mass independent terms really are $\mathrm{U}\left(2 N_{F}\right)$ invariant, and the mass dependent terms for the two cases are turned into each other.

$g_{R}$ can also be used to relate the two different embeddings of $\mathrm{SO}\left(2 N_{F}\right)$ in $\mathrm{SU}\left(2 N_{F}\right)$ to each other. For the Dirac case the $\mathrm{SO}(2 N)$ generators satisfied $Q^{a T} J_{S}=-J_{S} Q^{a}$ while for the Majorana case they satisfied $\tilde{Q}^{a T}=-\tilde{Q}^{a}$. The two sets of generators are related by

$$
\tilde{Q}^{a}=g_{R} Q^{a} g_{R}^{\dagger}, \quad \tilde{X}^{a}=g_{R} X^{a} g_{R}^{\dagger} .
$$




\section{$5 \quad$ Partially quenching and the quark flow technique}

A thorough discussion of $\mathrm{PQChPT}$ and in particular the derivation of the propagator used there is [49]. That discussion uses the supersymmetric method. Alternative methods of calculation are the replica trick [50] and the quark flow method [30]. The earliest partially quenched work for QCDlike theories used the supersymmetric method [23]. The replica trick has been used in [51]. We use the quark-flow method.

For this method we look at the matrix

$$
\Phi=\pi^{a} X^{a}
$$

for each of the cases.

For the QCD case, $\Phi$ is a traceless Hermitian matrix. We actually keep $\Phi$ in the flavour basis with elements $\phi_{i j}$ and $i, j$ are flavour indices. The tracelessness condition is enforced by the propagator. The indices are kept explicitly and the propagator connecting a field $\phi_{i j}$ to $\phi_{k l}$ is [49]

$$
G_{i j k l}(k)=G_{i j}^{c}(k) \delta_{i l} \delta_{j k}-\delta_{i j} \delta_{k l} G_{i k}^{q}(k) / n_{\text {sea }} .
$$

The number of sea quarks $n_{\text {sea }}$ is what we call $N_{F}$. with $G_{i j}^{c}=i /\left(p^{2}-\chi_{i j}\right)$. The neutral part of the propagator, $G_{i k}^{q}$, can contain double poles. In particular for the mass cases we consider:

$$
\begin{aligned}
G_{v v^{\prime}}^{q} & =i\left(\chi_{1}-\chi_{4}\right) /\left(p^{2}-\chi_{1}\right)^{2}+i /\left(p^{2}-\chi_{1}\right), \\
G_{v s}^{q} & =i /\left(p^{2}-\chi_{1}\right), \\
G_{s s^{\prime}}^{q} & =i /\left(p^{2}-\chi_{4}\right) .
\end{aligned}
$$

$v, s$ denote valence or sea quarks. The extra parts come from integrating out the $\Phi_{0}[49]$ and enforce the condition that $\Phi$ must be traceless. When constructing the Feynman diagrams, we keep all flavour indices free. Those that connect to external states get replaced by the value of the external valence flavour index and the remaining ones are summed over the sea quark flavours. In the present calculation, with all sea quarks the same mass, that corresponds to a factor of $N_{F}$ for each free flavour index.

For the Majorana, $\mathrm{SU}\left(N_{F}\right) \rightarrow \mathrm{SO}\left(N_{F}\right)$, case we have that $\Phi=\pi^{a} X^{a}$ with $\Phi$ Hermitian, traceless and symmetric. Hermitian and traceless follow from $\mathrm{SU}\left(N_{F}\right)$ and symmetric from (3.8). Going to the flavour basis for the diagonal elements of $\Phi$ there is no change w.r.t. the QCD case, but the flavour charged or off-diagonal elements must be correctly symmetrized. This has to be done both for the propagator and the connection to the external states, keeping track of the needed normalization. Afterwards we set the flavour indices connected to external states to their valence values and sum over the flavours for the free indices.

For the Dirac adjoint case, $\mathrm{SU}\left(2 N_{F}\right) \rightarrow \mathrm{SO}\left(2 N_{F}\right)$, case we have that $\Phi=\pi^{a} X^{a}$ with $\Phi$ Hermitian, traceless and satisfying $X^{a} J_{S}=J_{S} X^{a T}$ and the matrix $\Phi$ is $2 N_{F} \times 2 N_{F}$. Rewriting $\Phi$ with $N_{F} \times N_{F}$ matrices leads to the form

$$
\Phi=\left(\begin{array}{cc}
\Phi_{A} & \Phi_{C}^{\dagger} \\
\Phi_{C} & \Phi_{A}^{T}
\end{array}\right) \text {, with }\langle A\rangle=0, \Phi_{C}=\Phi_{C}^{T} .
$$


$\Phi_{A}$ is Hermitian. The elements in $\Phi_{A}$ correspond to quark-antiquark states, those in $\Phi_{C}$ to diquark states. $\Phi_{A}$ can be treated exactly as in the QCD case, both the diagonal and flavour charged or offdiagonal elements, since $\left\langle\Phi_{A}\right\rangle=0$ replaces $\langle\Phi\rangle=0$ in the QCD case. $\Phi_{C}$ can be treated as offdiagonal or flavour charged propagators but the needed symmetrizing should be taken care of both for external states and propagators. The normalization of all states must be done correctly as well. After constructing Feynman diagrams with both $\Phi_{A}$ and $\Phi_{C}$ degrees of freedom taken into account, we sum free index lines over the $N_{F}$ degrees of freedom, not $2 N_{F}$. The results always agree with the calculations done with the previous, Majorana, method.

For the last case, $\mathrm{SU}\left(2 N_{F}\right) \rightarrow \mathrm{Sp}\left(2 N_{F}\right)$, pseudo-real, we have that $\Phi=\pi^{a} X^{a}$ with $\Phi$ Hermitian, traceless and satisfying $X^{a} J_{A}=J_{A} X^{a T}$ and the matrix $\Phi$ is $2 N_{F} \times 2 N_{F}$. Rewriting $\Phi$ with $N_{F} \times N_{F}$ matrices leads to the form

$$
\Phi=\left(\begin{array}{ll}
\Phi_{A} & \Phi_{C}^{\dagger} \\
\Phi_{C} & \Phi_{A}^{T}
\end{array}\right), \text { with }\langle A\rangle=0, \Phi_{C}=-\Phi_{C}^{T} .
$$

$\Phi_{A}$ is Hermitian. The elements in $\Phi_{A}$ correspond to quark-antiquark states, those in $\Phi_{C}$ to diquark states. $\Phi_{A}$ can be treated exactly as in the QCD case, both the diagonal and flavour charged or offdiagonal elements, since $\left\langle\Phi_{A}\right\rangle=0$ replaces $\langle\Phi\rangle=0$ in the QCD case. $\Phi_{C}$ can be treated as offdiagonal or flavour charged propagators but the needed antisymmetrizing should be taken care of. The normalization of all states must be done correctly as well. After constructing Feynman diagrams with both $\Phi_{A}$ and $\Phi_{C}$ degrees of freedom taken into account, we sum free index lines over the $N_{F}$ degrees of freedom, not $2 N_{F}$. In this case and the previous we can also compare calculations with $\Phi_{A}$ or $\Phi_{C}$ external states providing a check on our results.

\section{Analytical results}

We have calculated the masses, decay constants and vacuum expectation values to NNLO for the QCD-like theories with the symmetry breaking patterns discussed above. A number of checks have been performed on the analytical formulas. The infinite volume unquenched results were obtained earlier in [24] and we have reproduced those. The partially quenched and finite volume results in the QCD case are finite. The partially quenched expressions reduce to the unquenched results whenever we set the sea mass equal to the valence mass. In addition we reproduce the known results at NLO for the condensate [23] also for the partially quenched case. The finite volume expressions have been checked against the known NLO results and numerically with the earlier known NNLO results, as discussed in section 7 .

For the real and pseudo-real case we have the additional check that calculating the mass or decay constant of a quark-anti-quark or a diquark meson gives the same results. This corresponds to using a field from the $A$ or the $C$ sector in the matrices (5.4), (5.5). For the real case we have the additional check that the results using the Dirac case and the Majorana case coincide. 
The finite volume case is always done for three spatial dimensions of size $\mathrm{L}$ and an infinite temporal volume. In addition we work in the center of mass system, the momenta are such that the external states have zero spatial momentum.

The masses are the physical masses defined as the pole of the full propagator. We consider here the case where all valence quarks have the same quark mass $m_{1}=\hat{m}$ and the sea quarks all have the same mass $m_{4}=m_{S}$. For the unquenced case obviously $m_{4}=m_{1}$. The labeling is similar to those used in three flavour PQChPT [31-33, 42]. In the formulas we use instead the quantities

$$
\chi_{1}=2 B_{0} m_{1}, \quad \chi_{4}=2 B_{0} \chi_{4}, \quad \chi_{14}=\frac{1}{2}\left(\chi_{1}+\chi_{4}\right) .
$$

These quantities are referred to in analyticalresults.txt (online resource 1) as m11, m44 and $\mathrm{m} 14$ respectively.

The formulas are given for the cases $\mathrm{SU}\left(N_{F}\right) \times \mathrm{SU}\left(N_{F}\right) \rightarrow \mathrm{SU}\left(N_{F}\right), \mathrm{SU}\left(N_{F}\right) \rightarrow$ $\mathrm{SO}\left(N_{F}\right)$ and $\mathrm{SU}\left(2 N_{F}\right) \rightarrow \mathrm{Sp}\left(2 N_{F}\right)$. Note the difference in convention for the second case compared to [24]. The three cases are referred to in the formulas with SUN, SON and SPN for the unquenced case and PQSUN,PQSON and PQSPN for the partially quenched case. In the latter case $N_{F}$ refers to the number of sea quarks.

For the mass we consider a meson made of a different quark and anti-quark or a diquark state with two different quarks. These are always valence quarks. The physical mass at finite volume is given by

$$
m_{\text {phys }}^{2}=\chi_{1}+m^{(4) 2}+\Delta^{V} m^{(4) 2}+m^{(6) 2}+\Delta^{V} m^{(6) 2} .
$$

The superscript $(n)$ labels the order $p^{n}$ correction and $\Delta^{V}$ indicates the finite volume corrections. In all cases the lowest order mass squared is given by $\chi_{1}$. A further break up is done for the LEC dependent parts via the $L_{i}^{r}$ (NLO) and $K_{i}^{r}$ (NNLO) and the remainder via

$$
\begin{aligned}
m^{(4) 2} & =m^{L(4) 2}+m^{R(4) 2} \\
m^{(6) 2} & =m^{K(6) 2}+m^{L(6) 2}+m^{R(6) 2} \\
\Delta^{V} m^{(6) 2} & =\Delta^{V} m^{L(6) 2}+\Delta^{V} m^{R(6) 2}
\end{aligned}
$$

All quantities are given explicitly in analyticalresults.txt (online resource 1).

The decay constant $F_{\text {phys }}$ for the same mesons as above is expanded w.r.t. the lowest order as

$$
F_{\text {phys }}=F_{\mathrm{LO}}\left(1+F^{(4)}+\Delta^{V} F^{(4)}+F^{(6) 2}+\Delta^{V} F^{(6)}\right)
$$

with a similar split

$$
\begin{aligned}
F^{(4)} & =F^{L(4)}+F^{R(4)} \\
F^{(6)} & =F^{K(6)}+F^{L(6)}+F^{R(6)} \\
\Delta^{V} F^{(6)} & =\Delta^{V} F^{L(6)}+\Delta^{V} F^{R(6)}
\end{aligned}
$$

All quantities are given explicitly in analyticalresults.txt (online resource 1). 
The vacuum expectation value is expanded in exactly the same way

$$
v_{\text {phys }}=v_{\mathrm{LO}}\left(1+v^{(4)}+\Delta^{V} v^{(4)}+v^{(6) 2}+\Delta^{V} v^{(6)}\right)
$$

with a similar split

$$
\begin{aligned}
v^{(4)} & =v^{L(4)}+v^{R(4)} \\
v^{(6)} & =v^{K(6)}+v^{L(4)}+v^{R(4)} \\
\Delta^{V} v^{(6)} & =\Delta^{V} v^{L(6)}+\Delta^{V} v^{R(6)}
\end{aligned}
$$

All quantities are given explicitly in analyticalresults.txt (online resource 1).

The quantities with $K$ for the SON and SPN case have been set to zero. They are polynomials up to the needed degree in $\chi_{1}$ and $\chi_{4}$, with an overall factor of $\chi_{1}$ for the mass.

The decay constant and the vacuum expectation value were defined implicitly in [24] using a generator $X^{a}$ in the axial current normalized to one and an element in $\hat{\mathcal{M}}$ normalized to one. The consequence was that in [24] $F_{\mathrm{LO}}=F$ and $v_{\mathrm{LO}}=-B_{0} F^{2}$ for all cases. This is not exactly what was done in earlier work leading to differences in factors of 2 and $\sqrt{2}$. Below we explicitly specify all definitions in terms of the quark fields.

QCD or complex representation. If we label the first Dirac (valence) quark by 1 and the second by 2 the decay constant and vacuum expectation value are defined as

$$
\begin{aligned}
\left\langle 0\left|\bar{q}_{1} \gamma_{\mu} \gamma_{5} q_{2}\right| M(p)\right\rangle & =i \sqrt{2} F_{\text {phys }} p_{\mu} \\
\left\langle\bar{q}_{1} q_{1}\right\rangle & =\left\langle\bar{q}_{L 1} q_{R 1}+\bar{q}_{R 1} q_{L 1}\right\rangle=v_{\text {phys }}
\end{aligned}
$$

$M$ denotes a meson of that quark content with momentum $p$.

The resulting lowest orders are

$$
F_{\mathrm{LO}}=F \quad v_{\mathrm{LO}}=-B_{0} F^{2}
$$

Adjoint or real representation. Here we have to be careful how we define the physical decay constant. We can choose to do using generators normalized to one using Dirac Fermions or generators normalized to one using the $\hat{q}_{i}$ elements.

With a Dirac fermion definition, the first Dirac (valence) quark labeled by 1 and the second by 2 , the definitions are

$$
\begin{aligned}
\left\langle 0\left|\bar{q}_{1} \gamma_{\mu} \gamma_{5} q_{2}\right| M(p)\right\rangle & =i \sqrt{2} F_{\text {phys }} p_{\mu} \\
\left\langle\bar{q}_{1} q_{1}\right\rangle & =\left\langle\bar{q}_{L 1} q_{R 1}+\bar{q}_{R 1} q_{L 1}\right\rangle=v_{\text {phys }}
\end{aligned}
$$

$M$ denotes a meson of that quark content with momentum $p$. The resulting lowest orders are

$$
F_{\mathrm{LO}}=\sqrt{2} F \quad v_{\mathrm{LO}}=-2 B_{0} F^{2} .
$$

If we instead choose to use the Majorana case, the natural definition of the decay constant and vacuum expectation value with the first (valence) Majorana fermion labeled 
as 1 and the second as 2 via

$$
\begin{aligned}
\frac{1}{2 \sqrt{2}}\left\langle 0\left|\hat{q}_{1}^{*} \bar{\sigma}_{\mu} \hat{q}_{2}+\hat{q}_{2}^{*} \bar{\sigma}_{\mu} \hat{q}_{1}\right| M(p)\right\rangle & =i \sqrt{2} F_{\text {phys }} p_{\mu} \\
\frac{1}{2}\left\langle\hat{q}_{1} \sigma^{2} \hat{q}_{1}+\hat{q}_{1}^{*} \sigma^{2} \hat{q}_{1}^{*}\right\rangle & =v_{\text {phys }}
\end{aligned}
$$

The resulting lowest orders are

$$
F_{\mathrm{LO}}=F \quad v_{\mathrm{LO}}=-B_{0} F^{2} .
$$

$N_{c}=2$ or pseudo-real representation. Here we again need to be careful how we define the physical decay constant. We can choose to do using generators normalized to one using the original Dirac Fermions or generators normalized to one using the $\hat{q}_{i}$ elements.

With a Dirac fermion definition, the first Dirac (valence) quark labeled by 1 and the second by 2 , the definitions are

$$
\begin{aligned}
\left\langle 0\left|\bar{q}_{1} \gamma_{\mu} \gamma_{5} q_{2}\right| M(p)\right\rangle & =i \sqrt{2} F_{\text {phys }} p_{\mu} \\
\left\langle\bar{q}_{1} q_{1}\right\rangle & =\left\langle\bar{q}_{L 1} q_{R 1}+\bar{q}_{R 1} q_{L 1}\right\rangle=v_{\text {phys }}
\end{aligned}
$$

$M$ denotes a meson of that quark content with momentum $p$. The resulting lowest orders are

$$
F_{\mathrm{LO}}=\sqrt{2} F \quad v_{\mathrm{LO}}=-2 B_{0} F^{2} .
$$

In terms of the $\hat{q}_{i}$ the definitions are

$$
\begin{aligned}
& \left\langle 0\left|\overline{\hat{q}}_{1} \gamma_{\mu} \overline{\hat{q}}_{2}+\overline{\hat{q}}_{1+N_{F}} \gamma_{\mu} \overline{\hat{q}}_{2+N_{F}}\right| M(p)\right\rangle=i \sqrt{2} F_{\text {phys }} p_{\mu} \\
& \frac{1}{2}\left\langle\overline{\hat{q}}_{1+N_{F}, a} \epsilon_{a b} C \overline{\hat{q}}_{1, b}-\overline{\hat{q}}_{1, a} \epsilon_{a b} C \overline{\hat{q}}_{1+N_{F}, b}-\hat{q}_{1+N_{F}, a} \epsilon_{a b} C \hat{q}_{1, b}+\hat{q}_{1, a} \epsilon_{a b} C \hat{q}_{1+N_{F}, b}\right\rangle=v_{\text {phys }} .
\end{aligned}
$$

\section{Numerical examples and checks}

The main aim of this work is to provide the lattice work with the formulas and programs needed to do the extrapolation to zero mass. We therefore only present some representative numerical results. The numerical programs are included in the latest version of CHIRON, [43, 44].

For the numbers presented we always use $\chi_{1}=0.14^{2} \mathrm{GeV}^{2}$, if not varied explicitly, and $F=0.0877 \mathrm{GeV}$ as well as a subtraction scale $\mu=0.77 \mathrm{GeV}$. The length $L$ for the finite volume has been chosen such that $L \times 0.14 \mathrm{GeV}=3$ or $L \approx 4.2 \mathrm{fm}$.

The LECs at NLO we choose to be those of the recent determination of [52] with the extra LEC $L_{0}^{r}=0$. The NNLO constants we have always put to zero.

A number of numerical checks for the QCD case have been done. The unquenched infinite volume results for three flavours agree with the three flavour results of $[53,54]$. The partially quenched results for masses and decay constants at infinite volume agree with the case $d_{\text {sea }}=1, d_{\text {val }}=1$ of [31-33]. The unquenched results for masses and decay constants at finite volume agree with [41]. The partially quenched results for masses and decay constants at finite volume agree with the case $d_{\text {sea }}=1, d_{\text {val }}=1$ of [42] and finally 
the unquenched finite volume results for the vacuum expectation value agree with the results of [38].

In figure 1 we show the mass squared for the infinite volume for all cases we have considered for three values of $N_{F}$. In general, as was already noticed in [24] the corrections are larger for the larger values of $N_{F}$. The corrections are also larger for the $\mathrm{SU}\left(2 N_{F}\right) \rightarrow$ $\operatorname{Sp}\left(2 N_{F}\right)$ case since this corresponds to a twice as large number of fermions as the other cases. The partially quenched results shown in the right column are at a fixed value of $\chi_{1}$. That explains why the corrections do not vanish for $\chi_{4}=0$.

The same types of results are shown for the decay constant in figure 2. The corrections are somewhat larger than for the masses but the convergence is typically somewhat better. The corrections for the vacuum expectation value shown in figure 3 are typically larger but with again a reasonable convergence from NLO to NNLO.

We can now make similar plots for the finite volume corrections. The overall size of them is as expected. The smallest $m L$ is about two for the left hand sides of all plots. In the unquenched case the exponential falloff with the mass is clearly visible. The partially quenched cases contain a fixed mass scale $\chi_{1}$ which is why the correction is more constant there, that mass stays at the $m L=3$ point for the plots. The dips are caused by the finite volume corrections going through zero. The corrections to the mass are shown in figure 4 , the decay constant in figure 5 and the vacuum expectation value in figure 6 .

\section{Conclusions}

We have calculated in the effective field theory for the three possible symmetry breaking patterns the NNLO order finite volume and partial quenching effects to NNLO in the expansion. The results satisfy a large number of checks agreeing analytically and numerically with earlier work that our results reduce to for some cases. The analytical part of this work relied heavily on FORM [55].

The analytical results are of reasonable length but given the total number of results we have included them as FORM output in a supplementary file (online resource 1). They can also be downloaded from [56].

The numerical programs have been included in CHIRON [43] version 0.54 which can be downloaded from [44]. We have presented results in a number of cases with typical QCD values of the parameters. The results are of the expected sizes from earlier work in three flavour ChPT. We hope these results will be useful for lattice studies of these alternative symmetry breaking patterns.

\section{Acknowledgments}

This work is supported in part by the Swedish Research Council grants 621-2011-5080 and 621-2013-4287. J.B. thanks the Centro de Ciencias de Benasque Pedro Pascual, where part of this work was done, for hospitality. 

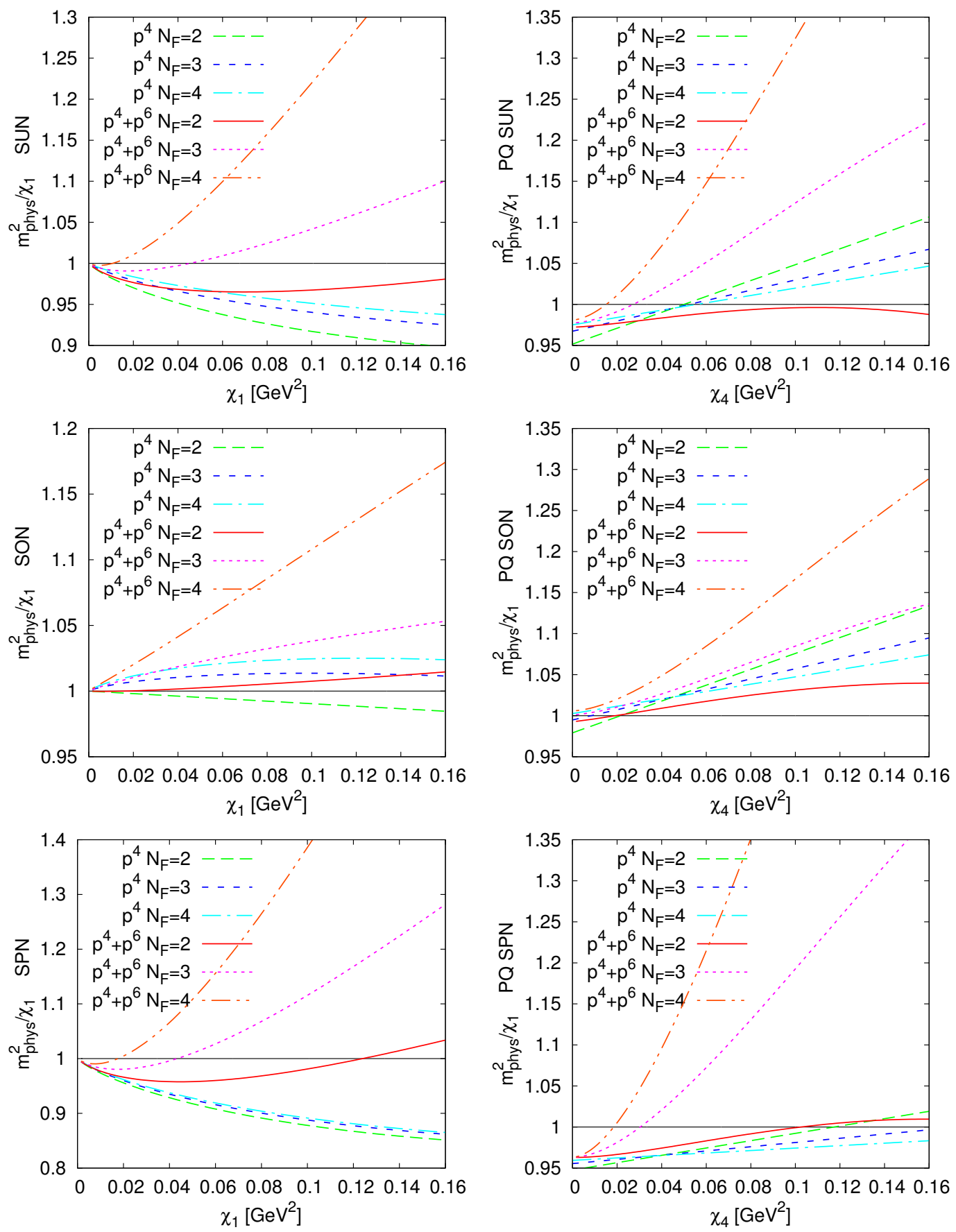

Figure 1. The physical mass squared divided by the lowest order mass squared for the unquenched (left) as a function of $\chi_{1}$ and the partially quenched case (right) as a function of $\chi_{4}$ with $\chi_{1}=$ $0.14^{2} \mathrm{GeV}^{2}$. Other input as in the text. Shown are the NLO $\left(p^{4}\right)$ and NNLO $\left(p^{4}+p^{6}\right)$ results for three values of $N_{F}$. Top line: $\mathrm{SU}\left(N_{F}\right) \times \mathrm{SU}\left(N_{F}\right) \rightarrow \mathrm{SU}\left(N_{F}\right)$. Middle line: $\mathrm{SU}\left(N_{F}\right) \rightarrow \mathrm{SO}\left(N_{F}\right)$. Bottom line: $\mathrm{SU}\left(2 N_{F}\right) \rightarrow \mathrm{Sp}\left(2 N_{F}\right)$. 

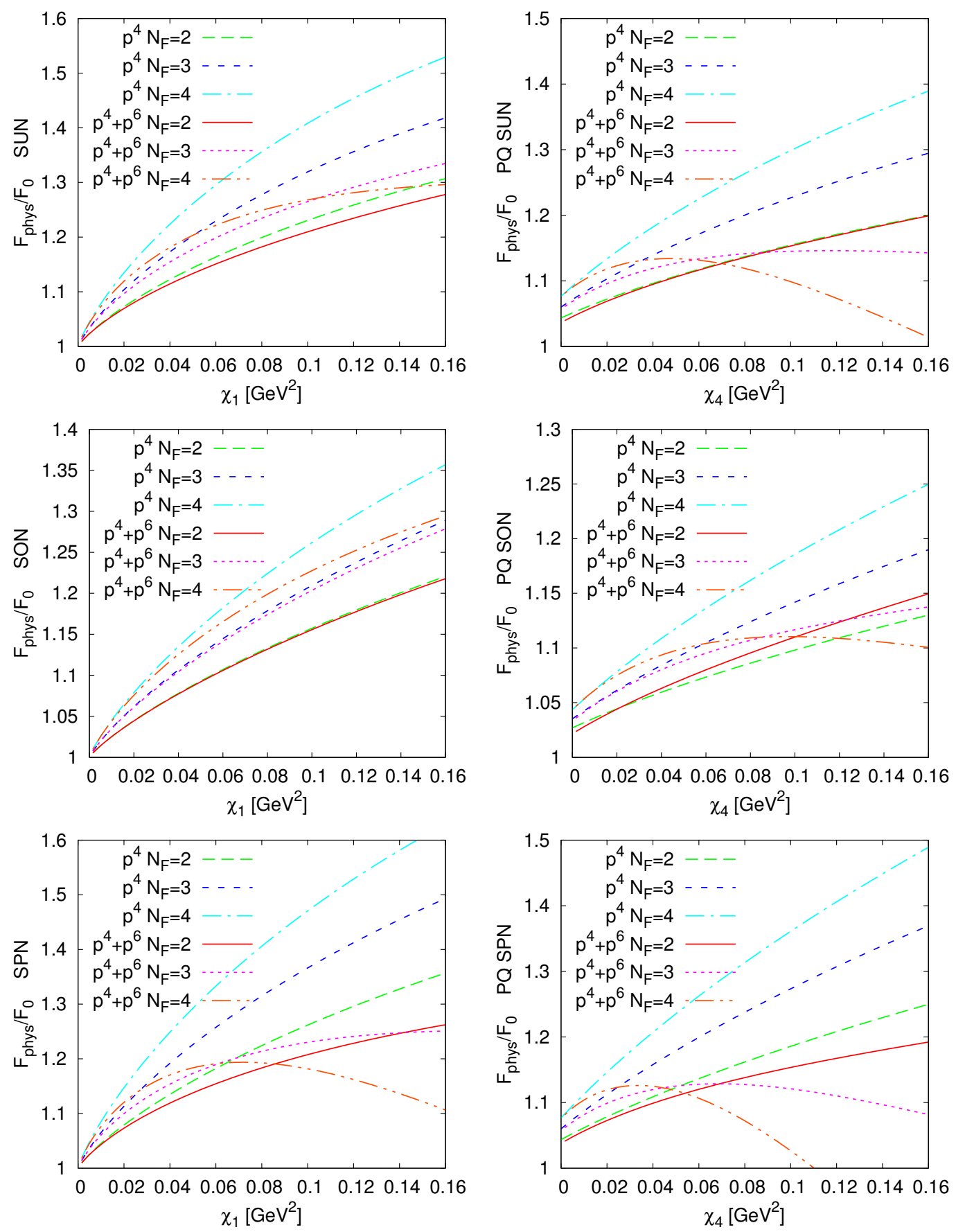

Figure 2. The decay constant divided by the lowest order value $F_{0}=F_{\mathrm{LO}}$ for the unquenched (left) as a function of $\chi_{1}$ and the partially quenched case (right) as a function of $\chi_{4}$ with $\chi_{1}=0.14^{2} \mathrm{GeV}^{2}$. Other input as in the text. Shown are the NLO $\left(p^{4}\right)$ and NNLO $\left(p^{4}+p^{6}\right)$ results for three values of $N_{F}$. Top line: $\mathrm{SU}\left(N_{F}\right) \times \mathrm{SU}\left(N_{F}\right) \rightarrow \mathrm{SU}\left(N_{F}\right)$. Middle line: $\mathrm{SU}\left(N_{F}\right) \rightarrow \mathrm{SO}\left(N_{F}\right)$. Bottom line: $\mathrm{SU}\left(2 N_{F}\right) \rightarrow \mathrm{Sp}\left(2 N_{F}\right)$. 

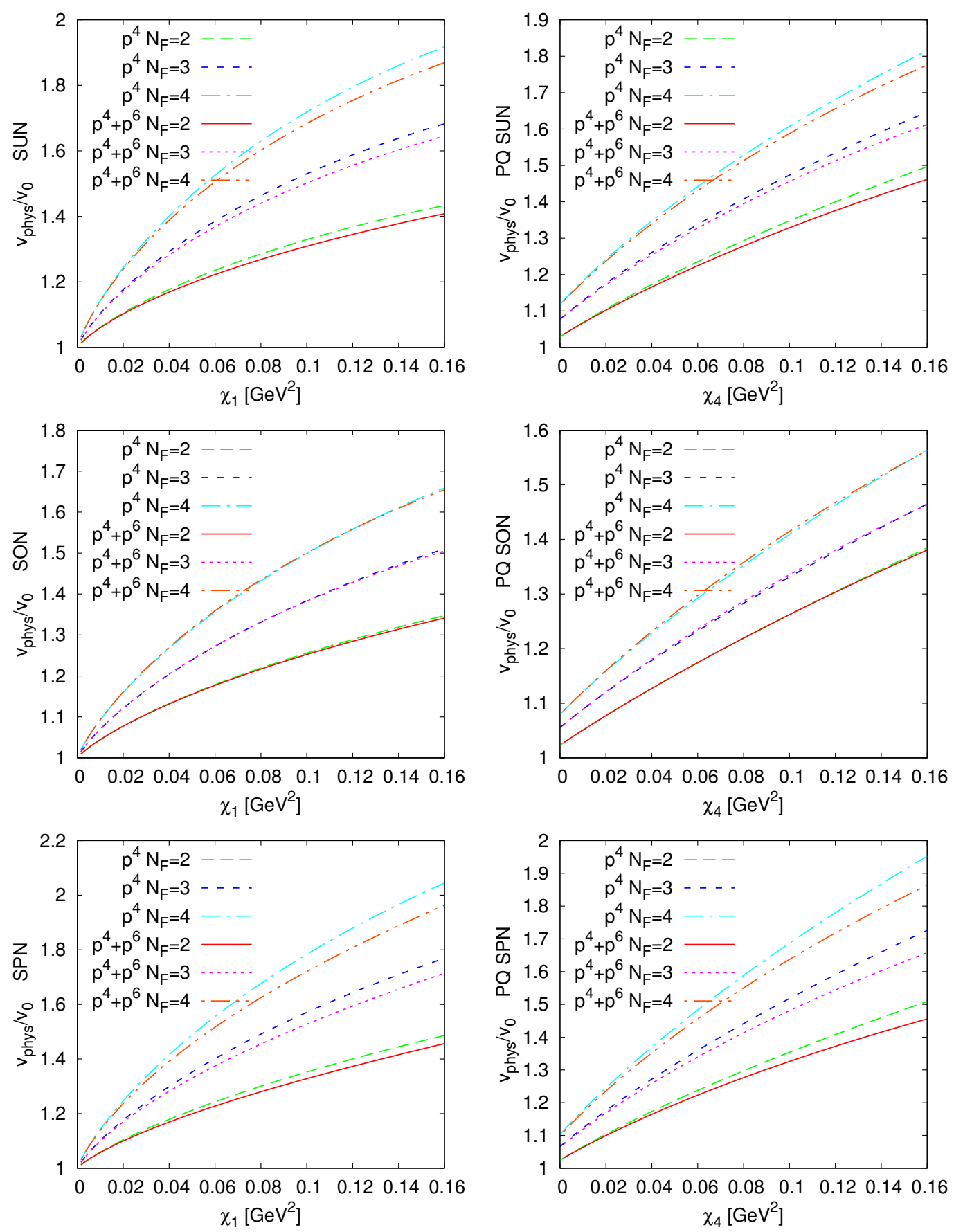

Figure 3. The vacuum expectation value divided by the lowest order value $v_{0}=v_{\mathrm{LO}}$ for the unquenched (left) as a function of $\chi_{1}$ and the partially quenched case (right) as a function of $\chi_{4}$ with $\chi_{1}=0.14^{2} \mathrm{GeV}^{2}$. Other input as in the text. Shown are the NLO $\left(p^{4}\right)$ and NNLO $\left(p^{4}+p^{6}\right)$ results for three values of $N_{F}$. Top line: $\mathrm{SU}\left(N_{F}\right) \times \mathrm{SU}\left(N_{F}\right) \rightarrow \mathrm{SU}\left(N_{F}\right)$. Middle line: $\mathrm{SU}\left(N_{F}\right) \rightarrow \mathrm{SO}\left(N_{F}\right)$. Bottom line: $\mathrm{SU}\left(2 N_{F}\right) \rightarrow \mathrm{Sp}\left(2 N_{F}\right)$. 

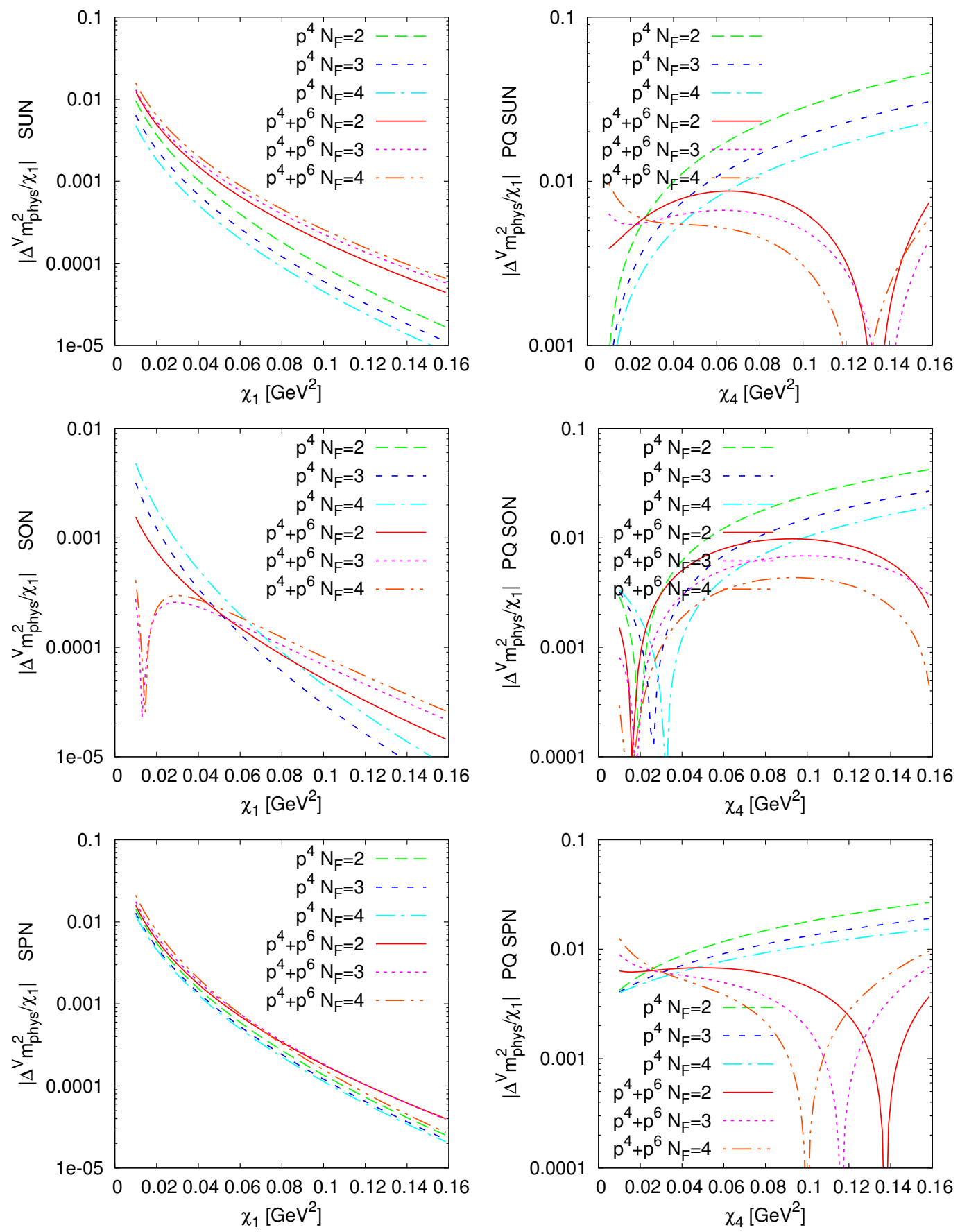

Figure 4. The absolute value of the finite volume correction to the physical mass squared divided by the lowest order mass squared for the unquenched (left) as a function of $\chi_{1}$ and the partially quenched case (right) as a function of $\chi_{4}$ with $\chi_{1}=0.14^{2} \mathrm{GeV}^{2}$. Shown are the NLO $\left(p^{4}\right)$ and NNLO $\left(p^{4}+p^{6}\right)$ results for three values of $N_{F}$. Top line: $\mathrm{SU}\left(N_{F}\right) \times \mathrm{SU}\left(N_{F}\right) \rightarrow \mathrm{SU}\left(N_{F}\right)$. Middle line: $\mathrm{SU}\left(N_{F}\right) \rightarrow \mathrm{SO}\left(N_{F}\right)$. Bottom line: $\mathrm{SU}\left(2 N_{F}\right) \rightarrow \mathrm{Sp}\left(2 N_{F}\right)$. 

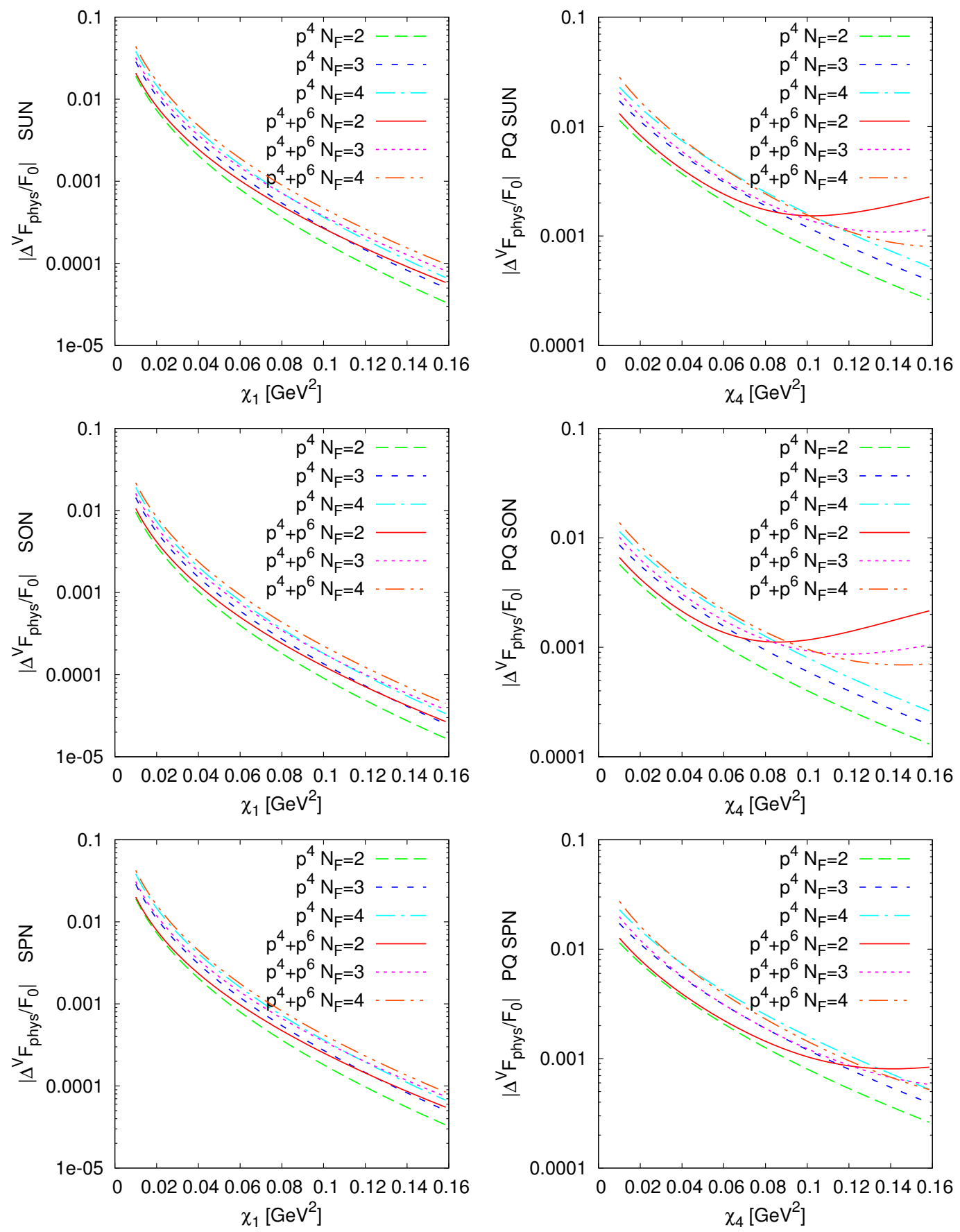

Figure 5. The absolute value of the finite volume correction to the decay constant divided by the lowest order value $F_{0}=F_{\mathrm{LO}}$ for the unquenched (left) as a function of $\chi_{1}$ and the partially quenched case (right) as a function of $\chi_{4}$ with $\chi_{1}=0.14^{2} \mathrm{GeV}^{2}$. Shown are the NLO $\left(p^{4}\right)$ and NNLO $\left(p^{4}+p^{6}\right)$ results for three values of $N_{F}$. Top line: $\mathrm{SU}\left(N_{F}\right) \times \mathrm{SU}\left(N_{F}\right) \rightarrow \mathrm{SU}\left(N_{F}\right)$. Middle line: $\mathrm{SU}\left(N_{F}\right) \rightarrow \mathrm{SO}\left(N_{F}\right)$. Bottom line: $\mathrm{SU}\left(2 N_{F}\right) \rightarrow \mathrm{Sp}\left(2 N_{F}\right)$. 

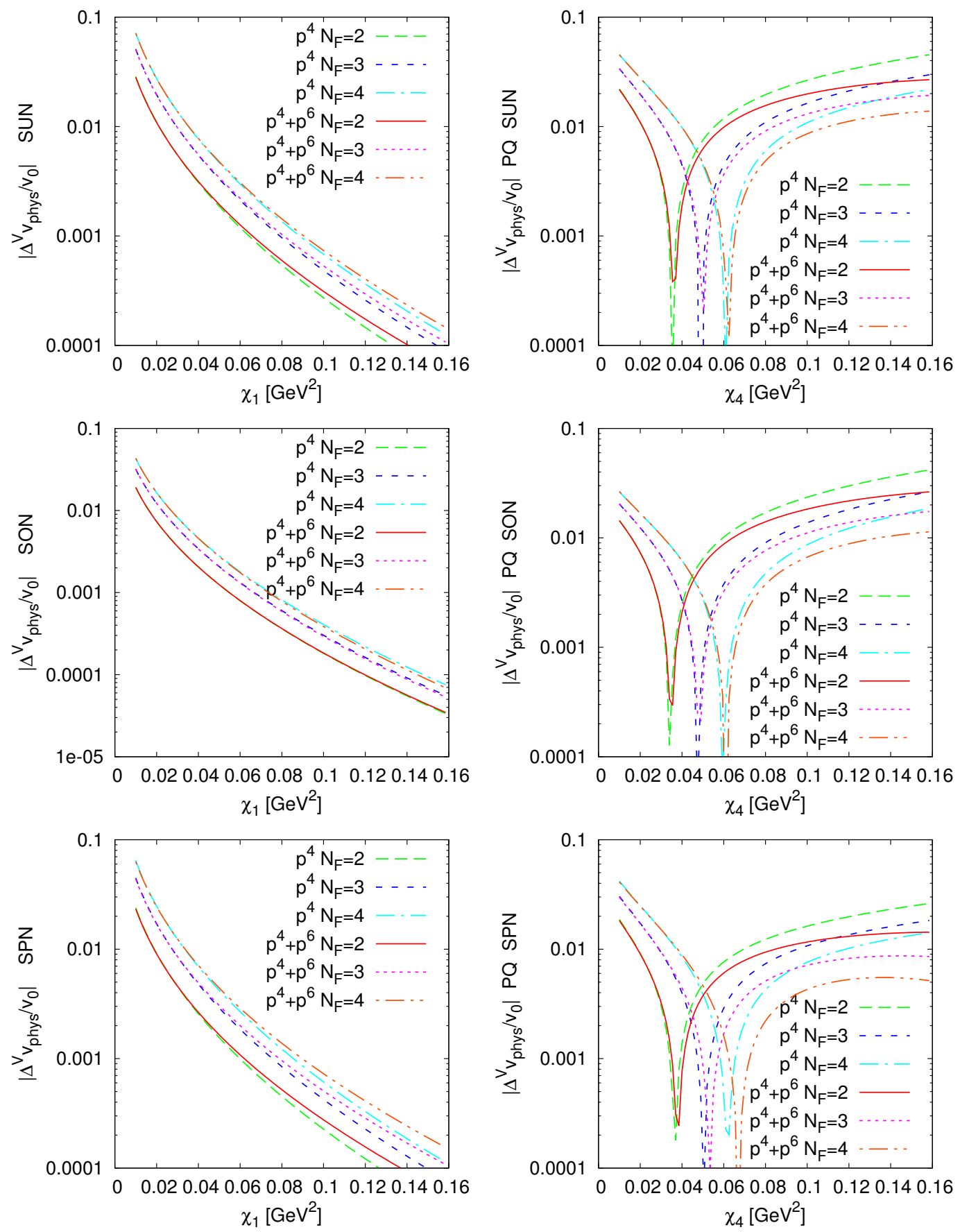

Figure 6. The absolute value of the finite volume correction to the vacuum expectation value divided by the lowest order value $v_{0}=v_{\mathrm{LO}}$ for the unquenched (left) as a function of $\chi_{1}$ and the partially quenched case (right) as a function of $\chi_{4}$ with $\chi_{1}=0.14^{2} \mathrm{GeV}^{2}$. Shown are the NLO $\left(p^{4}\right)$ and NNLO $\left(p^{4}+p^{6}\right)$ results for three values of $N_{F}$. Top line: $\mathrm{SU}\left(N_{F}\right) \times \mathrm{SU}\left(N_{F}\right) \rightarrow \mathrm{SU}\left(N_{F}\right)$. Middle line: $\mathrm{SU}\left(N_{F}\right) \rightarrow \mathrm{SO}\left(N_{F}\right)$. Bottom line: $\mathrm{SU}\left(2 N_{F}\right) \rightarrow \mathrm{Sp}\left(2 N_{F}\right)$. 
Open Access. This article is distributed under the terms of the Creative Commons Attribution License (CC-BY 4.0), which permits any use, distribution and reproduction in any medium, provided the original author(s) and source are credited.

\section{References}

[1] N. Brambilla et al., QCD and Strongly Coupled Gauge Theories: Challenges and Perspectives, Eur. Phys. J. C 74 (2014) 2981 [arXiv:1404.3723] [InSPIRE].

[2] F. Sannino, Lattice's Bright, Dark and Safe side, plenary talk at at the 33rd International Symposium on Lattice Field Theory.

[3] A. Hasenfratz, Beyond the Standard Model: lattice calculations at the energy frontier, plenary talk at the 33rd International Symposium on Lattice Field Theory.

[4] J. Kuti, The Higgs particle and the lattice, PoS (LATTICE 2013) 004.

[5] R. Lewis, C. Pica and F. Sannino, Light Asymmetric Dark Matter on the Lattice: SU(2) Technicolor with Two Fundamental Flavors, Phys. Rev. D 85 (2012) 014504 [arXiv: 1109.3513] [INSPIRE].

[6] A. Hietanen, R. Lewis, C. Pica and F. Sannino, Fundamental Composite Higgs Dynamics on the Lattice: SU(2) with Two Flavors, JHEP 07 (2014) 116 [arXiv:1404.2794] [INSPIRE].

[7] LSD collaboration, D. Schaich et al., Finite-temperature study of eight-flavor SU(3) gauge theory, arXiv:1506.08791 [INSPIRE].

[8] T.N. da Silva, E. Pallante and L. Robroek, Conformal or Confining, arXiv:1506.06396 [INSPIRE].

[9] M.P. Lombardo, K. Miura, T.J. Nunes da Silva and E. Pallante, One, two, zero: Scales of strong interactions, Int. J. Mod. Phys. A 29 (2014) 1445007 [arXiv:1410.2036] [InSPIRE].

[10] T. Appelquist et al., WW Scattering Parameters via Pseudoscalar Phase Shifts, Phys. Rev. D 85 (2012) 074505 [arXiv: 1201.3977] [INSPIRE].

[11] T. DeGrand, Y. Liu, E.T. Neil, Y. Shamir and B. Svetitsky, Spectroscopy of SU(4) gauge theory with two flavors of sextet fermions, Phys. Rev. D 91 (2015) 114502 [arXiv: 1501.05665] [INSPIRE].

[12] J.R. Andersen et al., Discovering Technicolor, Eur. Phys. J. Plus 126 (2011) 81 [arXiv:1104.1255] [INSPIRE].

[13] F. Sannino, Conformal Dynamics for TeV Physics and Cosmology, Acta Phys. Polon. B 40 (2009) 3533 [arXiv: 0911.0931] [INSPIRE].

[14] C.T. Hill and E.H. Simmons, Strong dynamics and electroweak symmetry breaking, Phys. Rept. 381 (2003) 235 [Erratum ibid. 390 (2004) 553] [hep-ph/0203079] [INSPIRE].

[15] S. Weinberg, Phenomenological Lagrangians, Physica A 96 (1979) 327 [InSPIRE].

[16] J. Gasser and H. Leutwyler, Chiral Perturbation Theory to One Loop, Annals Phys. 158 (1984) 142 [INSPIRE].

[17] J. Gasser and H. Leutwyler, Chiral Perturbation Theory: Expansions in the Mass of the Strange Quark, Nucl. Phys. B 250 (1985) 465 [INSPIRE].

[18] M.E. Peskin, The Alignment of the Vacuum in Theories of Technicolor, Nucl. Phys. B 175 (1980) 197 [INSPIRE]. 
[19] J. Preskill, Subgroup Alignment in Hypercolor Theories, Nucl. Phys. B 177 (1981) 21 [INSPIRE].

[20] S. Dimopoulos, Technicolored Signatures, Nucl. Phys. B 168 (1980) 69 [InSPIRE].

[21] J.B. Kogut, M.A. Stephanov, D. Toublan, J.J.M. Verbaarschot and A. Zhitnitsky, QCD-like theories at finite baryon density, Nucl. Phys. B 582 (2000) 477 [hep-ph/0001171] [INSPIRE].

[22] K. Splittorff, D. Toublan and J.J.M. Verbaarschot, Diquark condensate in QCD with two colors at next-to-leading order, Nucl. Phys. B 620 (2002) 290 [hep-ph/0108040] [INSPIRE].

[23] D. Toublan and J.J.M. Verbaarschot, The Spectral density of the QCD Dirac operator and patterns of chiral symmetry breaking, Nucl. Phys. B 560 (1999) 259 [hep-th/9904199] [INSPIRE].

[24] J. Bijnens and J. Lu, Technicolor and other QCD-like theories at next-to-next-to-leading order, JHEP 11 (2009) 116 [arXiv:0910.5424] [INSPIRE].

[25] J. Bijnens and J. Lu, Meson-meson Scattering in QCD-like Theories, JHEP 03 (2011) 028 [arXiv:1102.0172] [INSPIRE].

[26] J. Bijnens and J. Lu, Two-Point Functions and S-Parameter in QCD-like Theories, JHEP 01 (2012) 081 [arXiv:1111.1886] [INSPIRE].

[27] C.W. Bernard and M.F.L. Golterman, Partially quenched gauge theories and an application to staggered fermions, Phys. Rev. D 49 (1994) 486 [hep-lat/9306005] [INSPIRE].

[28] C. Bernard and M. Golterman, On the foundations of partially quenched chiral perturbation theory, Phys. Rev. D 88 (2013) 014004 [arXiv: 1304.1948] [INSPIRE].

[29] S.R. Sharpe and N. Shoresh, Physical results from unphysical simulations, Phys. Rev. D 62 (2000) 094503 [hep-lat/0006017] [INSPIRE].

[30] S.R. Sharpe, Quenched chiral logarithms, Phys. Rev. D 46 (1992) 3146 [hep-lat/9205020] [INSPIRE].

[31] J. Bijnens, N. Danielsson and T.A. Lähde, The Pseudoscalar meson mass to two loops in three-flavor partially quenched chiral perturbation theory, Phys. Rev. D 70 (2004) 111503 [hep-lat/0406017] [INSPIRE].

[32] J. Bijnens and T.A. Lähde, Decay constants of pseudoscalar mesons to two loops in three-flavor partially quenched chiral perturbation theory, Phys. Rev. D 71 (2005) 094502 [hep-lat/0501014] [INSPIRE].

[33] J. Bijnens, N. Danielsson and T.A. Lähde, Three-flavor partially quenched chiral perturbation theory at NNLO for meson masses and decay constants, Phys. Rev. D 73 (2006) 074509 [hep-lat/0602003] [INSPIRE].

[34] J. Gasser and H. Leutwyler, Light Quarks at Low Temperatures, Phys. Lett. B 184 (1987) 83 [INSPIRE].

[35] J. Gasser and H. Leutwyler, Thermodynamics of Chiral Symmetry, Phys. Lett. B 188 (1987) 477 [INSPIRE].

[36] J. Gasser and H. Leutwyler, Spontaneously Broken Symmetries: Effective Lagrangians at Finite Volume, Nucl. Phys. B 307 (1988) 763 [InSPIRE].

[37] G. Colangelo and C. Haefeli, Finite volume effects for the pion mass at two loops, Nucl. Phys. B 744 (2006) 14 [hep-lat/0602017] [INSPIRE]. 
[38] J. Bijnens and K. Ghorbani, Finite volume dependence of the quark-antiquark vacuum expectation value, Phys. Lett. B 636 (2006) 51 [hep-lat/0602019] [INSPIRE].

[39] P.H. Damgaard and H. Fukaya, The Chiral Condensate in a Finite Volume, JHEP 01 (2009) 052 [arXiv:0812.2797] [INSPIRE].

[40] J. Bijnens, E. Boström and T.A. Lähde, Two-loop Sunset Integrals at Finite Volume, JHEP 01 (2014) 019 [arXiv:1311.3531] [INSPIRE].

[41] J. Bijnens and T. Rössler, Finite Volume at Two-loops in Chiral Perturbation Theory, JHEP 01 (2015) 034 [arXiv: 1411.6384] [INSPIRE].

[42] J. Bijnens and T. Rössler, Finite Volume for Three-Flavour Partially Quenched Chiral Perturbation Theory through NNLO in the Meson Sector, arXiv:1508.07238 [INSPIRE].

[43] J. Bijnens, CHIRON: a package for ChPT numerical results at two loops, Eur. Phys. J. C 75 (2015) 27 [arXiv: 1412.0887] [INSPIRE].

[44] J. Bijnens, CHIRON: numerical tools for Chiral Perturbation Theory, http://www.thep.lu.se/ bijnens/chiron/.

[45] J. Bijnens, G. Colangelo and G. Ecker, The Mesonic chiral Lagrangian of order $p^{6}$, JHEP 02 (1999) 020 [hep-ph/9902437] [inSPIRE].

[46] J. Bijnens, G. Colangelo and G. Ecker, Renormalization of chiral perturbation theory to order $p^{6}$, Annals Phys. 280 (2000) 100 [hep-ph/9907333] [INSPIRE].

[47] S.R. Coleman, J. Wess and B. Zumino, Structure of phenomenological Lagrangians. 1., Phys. Rev. 177 (1969) 2239 [INSPIRE].

[48] C.G. Callan Jr., S.R. Coleman, J. Wess and B. Zumino, Structure of phenomenological Lagrangians. 2., Phys. Rev. 177 (1969) 2247 [INSPIRE].

[49] S.R. Sharpe and N. Shoresh, Partially quenched chiral perturbation theory without $\Phi_{0}$, Phys. Rev. D 64 (2001) 114510 [hep-lat/0108003] [INSPIRE].

[50] P.H. Damgaard and K. Splittorff, Partially quenched chiral perturbation theory and the replica method, Phys. Rev. D 62 (2000) 054509 [hep-lat/0003017] [INSPIRE].

[51] J. Levinsen, Finite volume chiral partition functions and the replica method, Phys. Rev. D 67 (2003) 125009 [hep-th/0301008] [INSPIRE].

[52] J. Bijnens and G. Ecker, Mesonic low-energy constants, Ann. Rev. Nucl. Part. Sci. 64 (2014) 149 [arXiv: 1405.6488] [INSPIRE].

[53] G. Amorós, J. Bijnens and P. Talavera, Two point functions at two loops in three flavor chiral perturbation theory, Nucl. Phys. B 568 (2000) 319 [hep-ph/9907264] [INSPIRE].

[54] G. Amorós, J. Bijnens and P. Talavera, $K_{\ell 4}$ form-factors and $\Pi-\Pi$ scattering, Nucl. Phys. B $\mathbf{5 8 5}$ (2000) 293 [Erratum ibid. B 598 (2001) 665] [hep-ph/0003258] [INSPIRE].

[55] J.A.M. Vermaseren, New features of FORM, math-ph/0010025 [INSPIRE].

[56] J. Bijnens, Chiral Perturbation Theory, http://www.thep.lu.se/ bijnens/chpt/. 